\title{
ALGORITHMS FOR TRANSLATIONAL TILING
}

\author{
MIHAIL N. KOLOUNTZAKIS \& MÁTÉ MATOLCSI
}

\begin{abstract}
In this paper we study algorithms for tiling problems. We show that the conditions (T1) and (T2) of Coven and Meyerowitz [5], conjectured to be necessary and sufficient for a finite set $A$ to tile the integers, can be checked in time polynomial in $\operatorname{diam}(A)$. We also give heuristic algorithms to find all non-periodic tilings of a cyclic group $\mathbb{Z}_{N}$. In particular we carry out a full classification of all non-periodic tilings of $\mathbb{Z}_{144}$.
\end{abstract}

2000 Mathematics Subject Classification: 05B45, 43A25, 68W30, 68T20.

Keywords and phrases. Translational tiles, algorithms

\section{TransLational TILING}

Definition 1.1. We say that a subset $A$ of an abelian group $G$ (written additively) tiles $G$ by translation when there exists a subset $B \subset G$ such that every $g \in G$ has a unique representation

$$
g=a+b, \quad(a \in A, b \in B) .
$$

This situation is denoted by $G=A \oplus B$ or $G=B \oplus A$. The sets $A$ and $B$ will be called tiling complements of each other.

Remark 1.1. In much of the bibliography, especially the earliest one and that with a more algebraic focus, the tiling condition $G=A \oplus B$ is often called a factorization of the group $G$.

In this paper we are primarily interested in the case when $G$ is a finite group, typically a cyclic group $\mathbb{Z}_{N}=\mathbb{Z} /(N \mathbb{Z})$, or the group $\mathbb{Z}^{d}, d \geq 1$.

\subsection{Periodicity.}

Definition 1.2. A set $B \subseteq G$ is called periodic if there exists a nonzero $g \in G$ such that $B+g=B$. Such a $g$ is then called a period of $B$ and clearly the set of all periods plus 0 forms a subgroup of $G$.

In case $B \subseteq \mathbb{Z}$ is a periodic set of integers, its least positive period is denoted by $\mathcal{P}(B)$.

A periodic set $B$ is clearly a union of congruence classes $\bmod \mathcal{P}(B)$ and we can write

$$
B=\widetilde{B} \oplus \mathcal{P}(B) \mathbb{Z},
$$

where $\widetilde{B} \subseteq[\mathcal{P}(B) \mathbb{Z}]$ and we have used the notation

$$
[n]=\{0,1, \ldots, n-1\} .
$$

It has long been known [20] that if $A$ is a finite set of integers and $\mathbb{Z}=A \oplus B$ is a tiling of the integers then the set $B \subseteq \mathbb{Z}$ is necessarily periodic. A consequence of this is that the study of any such tiling with period, say, $N=\mathcal{P}(B)$, reduces to the study of a tiling of the group $\mathbb{Z}_{N}$, namely the tiling $\mathbb{Z}_{N}=A \oplus \widetilde{B}$.

Date: October 2008.

MK: Supported by research grant No 2569 from the Univ. of Crete.

MM: Supported by Hungarian National Foundation for Scientific Research (OTKA), Grants No. PF64061, T-049301, T-047276. 
Non-periodic tilings of a group $G=A \oplus B$ are in some sense less structured and more interesting. Indeed, if $h \in G \backslash\{0\}$ is a period of $B$ and $H \leq G$ is the subgroup generated by $h$, then we can write $B=B^{\prime} \oplus H$ for some $B^{\prime} \subseteq G / H$ (slight, harmless abuse of language here). The tiling condition $G=A \oplus B$ then becomes equivalent to the tiling condition $G / H=A \oplus B^{\prime}$. This means that periodicity implies an immediate reduction to the complexity of the problem. Hajós [12] called a finite abelian group $G$ a good group if in any tiling $G=A \oplus B$ one of the two sets $A$ and $B$ must be periodic. Sands [23, 24] completed the classification of all finite abelian groups into good and bad groups (for the entire list see also [19, Prop. 4.1]). The smallest cyclic group that is not good is $\mathbb{Z}_{72}$.

1.2. Tiling in Fourier space. Cyclotomic polynomials. The condition $G=A \oplus B$ is clearly equivalent to

$$
\sum_{g \in G} \chi_{A}(g) \chi_{B}(x-g)=1, \quad(x \in G),
$$

which we can rewrite as $\chi_{A} * \chi_{B}=1$, with $*$ denoting the convolution of two functions.

Assume for simplicity that $G$ is finite from now on. For a function $f: G \rightarrow \mathbb{C}$ its Fourier Transform [22] is defined as the function $\widehat{f}: \Gamma \rightarrow \mathbb{C}$ given by

$$
\widehat{f}(\gamma)=\sum_{g \in G} f(g) \overline{\gamma(g)},
$$

$\Gamma$ being the dual group of $G$. It consists of all characters $\gamma$ which are the group homomorphisms $G \rightarrow \mathbb{C}$. It is easy to see that $\widehat{f * g}=\widehat{f} \cdot \widehat{g}$. Using this our tiling condition (1) can be rewritten as

$$
\widehat{\chi_{A}} \cdot \widehat{\chi_{B}}=\widehat{1}=|G| \chi_{\{0\}} .
$$

This is in turn equivalent to the obvious condition $|A| \cdot|B|=|G|$ as well as

$$
Z\left(\widehat{\chi_{A}}\right) \cup Z\left(\widehat{\chi_{B}}\right)=G \backslash\{0\},
$$

where $Z(f)=\{x: f(x)=0\}$ is the zero set of $f$. So, to check that two sets $A, B \subset G$ are tiling complements we have to first check the obvious condition $|A| \cdot|B|=|G|$ and verify that the zero sets cover everything but 0 .

In the particular case where $G=\mathbb{Z}_{N}$ is a cyclic group, we have that $\Gamma$ is isomorphic to $G$ and the FT of $f: G \rightarrow \mathbb{C}$ now becomes the function on $\mathbb{Z}_{N}$

$$
\widehat{f}(k)=\sum_{j=0}^{N-1} f(j) e^{-2 \pi i k j / N}, \quad(k=0,1, \ldots, N-1) .
$$

Introducing the polynomial $P(X)=\sum_{j=0}^{N-1} f(j) X^{j}$ it is often convenient to view the FT $\widehat{f}$ as the evaluation of $P(X)$ at the $N$-th roots of unity

$$
1, e^{2 \pi i \frac{1}{N}}, e^{-2 \pi i \frac{2}{N}}, \ldots, e^{-2 \pi i \frac{N-1}{N}} .
$$

It is the roots of this polynomial $P(X)$ that matter when one checks condition (2). And in the case where $f=\chi_{A}$ is the indicator function of a set $A \subseteq \mathbb{Z}_{N}$ the polynomial $P(X)$ has integer coefficients. The irreducible (over $\mathbb{Z}$ ) factors of $P(X)$ which are responsible for the zeros of $P(X)$ at $N$-th roots of unity are cyclotomic polynomials, which are defined as the minimal polynomials of roots of unity (see [5] for a brief introduction to the cyclotomic polynomials).

A quick way to define the cyclotomic polynomials $\Phi_{n}(X)$ is by the decomposition of $X^{N}-1$ into irreducible factors

$$
X^{N}-1=\prod_{d \mid N} \Phi_{d}(X)
$$

where the product extends over all divisors of $N, 1$ and $N$ included. 
The irreducible monic polynomial $\Phi_{n}(X)$ has as roots precisely the primitive $n$-th roots of unity, which are therefore algebraic conjugates of each other

$$
\Phi_{n}(X)=\prod_{0 \leq k<n,(k, n)=1}\left(X-e^{2 \pi i k / n}\right) .
$$

As a consequence, whenever an integer polynomial vanishes on a primitive $n$-th root of unity it vanishes on all of them. When vanishing of such a polynomial is the issue, therefore, the set of $N$-th roots of unity is split into $d(N)$ (= number of divisors of $N$ ) blocks, and the zero set of such polynomial $P(X)$ among the roots of unity is a union of such cyclotomic blocks.

Remark 1.2. Let us also observe that periodicity of a set $A \subseteq \mathbb{Z}_{N}$ can easily be detected on the Fourier side. Indeed, it is easy to see that, if $N=a b$, the set $A \subseteq \mathbb{Z}_{N}$ is a-periodic if and only if its Fourier transform $\widehat{\chi_{A}}(k)$ vanishes on all $k \in\{0,1, \ldots, N-1\}$ which are not multiples of $b$.

1.3. The Coven-Meyerowitz conditions. Suppose $A$ is a finite set of nonnegative integers (we assume $0 \in A$ ) and write, as is customary,

$$
A(X)=\sum_{a \in A} X^{a} .
$$

Let $S_{A}$ be the set of prime powers $p^{a}$ such that $\Phi_{p^{a}}(X) \mid A(X)$. In [5] Coven and Meyerowitz wrote down the following two conditions on a such a polynomial $A(X)$.

$\left(T_{1}\right) A(1)=\prod_{s \in S_{A}} \Phi_{s}(1)$,

$\left(T_{2}\right)$ If $s_{1}, \ldots, s_{m} \in S_{A}$ are powers of distinct primes then $\Phi_{s_{1} \cdots s_{m}}(X) \mid A(X)$.

They proved in [5] that if $\left(T_{1}\right)$ and $\left(T_{2}\right)$ hold for a set $A$ then $A$ tiles the integers by translation. Another way to say this is that there is a number $N$ such that $A$ tiles $\mathbb{Z}_{N}$. In the converse direction they proved that $\left(T_{1}\right)$ necessarily holds for any tile $A$. Regarding the necessity of $\left(T_{2}\right)$ for tiling it was proved in [5] that $\left(T_{2}\right)$ is also necessary for tiling when $|A|$ has at most two different prime factors. It was conjectured by Konyagin and Eaba [14] that $A$ is a tile of the integers if and only if both $\left(T_{1}\right)$ and $\left(T_{2}\right)$ hold.

1.4. The computational status of tiling. The most basic computational problem of tiling is to decide whether a given finite set $A$ in an abelian group $G$ tiles the group. If one ignores, as a first approach, questions of complexity and restricts oneself to questions of decidability, one must assume $G$ to be infinite (and, of course, discrete) for the problem to be meaningful.

In a more general form of the problem, that of asking whether a given set of tiles can be moved around (by a group of motions) to tile $\mathbb{R}^{d}$, tiling has long been shown to be undecidable. Berger 4 first showed this (it is undecidable to determine if a given finite set of polygons can tile $\mathbb{R}^{2}$ using rigid motions). Many other models of tiling have been shown to undecidable.

It is the case of tiling by translations and by a single tile that interests us here.

When the group $G$ is the group of integers $\mathbb{Z}$ the problem is decidable. This nontrivial fact follows from Newman's result [20] that every translational tiling of $\mathbb{Z}$ by a finite set is periodic. Although a bound for the period $\left(2^{\operatorname{diam} A}\right)$ is given by Newman's theorem, this is not strictly necessary to deduce the mere existence of an algorithm to decide tiling by $A$ (if, that is, we do not care about the running time of the algorithm). Indeed, given the finite $A \subset \mathbb{Z}$, all we have to do is to start examining, by exhaustive search, whether $A$ can tile the set $\{-N, \ldots, N\}$ for larger and larger $N$. Tiling here means that we want to find a collection of non-overlapping translates of $A$ which will cover $\{-N, \ldots, N\}$. If the set $A$ cannot tile $\mathbb{Z}$ then there is a finite $N$ for which we will not be able to tile $\{-N, \ldots, N\}$. This is a simple compactness (or diagonalization) argument. On the other hand, if $A$ does 
tile $\mathbb{Z}$ then, for large enough $N$, we will observe a tiling of $\{-N, \ldots, N\}$ which is "periodic" and which can, therefore, be extended indefinitely to the left and right to tile $\mathbb{Z}$.

The argument using periodicity given above is very general (see the introduction of [21], where it is also proved that if a set admits a tiling of the plane with one period then it also admits a fully periodic tiling), works in all dimensions, and it is enough that periodic tilings exist. It is not necessary for all tilings to be periodic (which fails to be the case even in dimension 2 for as simple a tile as a rectangle). Thus, the so-called periodic tiling conjecture [11, 18] (everything that tiles by translation can also tile periodically) implies decidability of translational tiling. This conjecture is still open in all dimensions $d \geq 2$.

Already when the group is $\mathbb{Z}^{2}$ the question of deciding if a given finite set $A \subset \mathbb{Z}^{2}$ can tile by translation is wide open, apart from the result of Szegedy [26] who gave an algorithm for the special cases of $|A|$ being a prime or 4 . There are also algorithms for other special cases but these all have topological conditions [28, 10] on the tile (e.g. to be simply connected).

Let us now restrict ourselves to the decision problem of deciding whether a given $A \subseteq \mathbb{Z}_{N}$ can tile $\mathbb{Z}_{N}$ by translation. We are interested to study the computational complexity of this problem regarding $N$ as the parameter. In particular we'd like to have an algorithm which runs in time $O\left(N^{c}\right)$ for some fixed $c>0$. Such an algorithm is still lacking though except when some arithmetic conditions on $|A|$ are imposed. In this paper we prove that if $|A|$ has at most two prime factors then we can decide if $A$ is a tile of $\mathbb{Z}$ in polynomial time. This is so because we can decide the Coven-Meyerowitz conditions $\left(T_{1}\right)$ and $\left(T_{2}\right)$ (see $\$ 1.3$ in polynomial time (Theorem 2.1 below). We also introduce a local version of the Coven-Meyerowitz conditions in $\S 3$ which will allow us to give a similar algorithm, under the same arithmetic conditions, for when $A$ tiles $\mathbb{Z}_{N}$.

On the other hand, some very similar problems to the decision of tiling (problem DIFF in [13]) have been shown to be NP-complete. This would suggest a lower bound in the computational complexity of the tiling decision problem. Were this decision problem to prove to be NP complete this would refute the equivalence of $\left(T_{1} \& T_{2}\right)$ to tiling (conjectured in [14]), assuming of course $\mathrm{P} \neq \mathrm{NP}$.

In the last section of the paper we turn to the problem of finding all non-periodic tilings of a cyclic group $\mathbb{Z}_{N}$. As explained in subsection 1.1, periodic tilings are less interesting because they can be considered as tilings of factor groups of $\mathbb{Z}_{N}$. Finding many (or, indeed all) non-periodic tilings of $\mathbb{Z}_{N}$ could be a way of testing the Coven-Meyerowitz conditions and possibly producing counterexamples. Besides being mathematically interesting on its own right, this problem has a particular motivation in certain modern music compositions. The interaction of mathematical theory and musical background has been extensively studied in recent years [1, 2, 3, 7, 27.

H. Fripertinger listed out all non-periodic tilings of $\mathbb{Z}_{72}$ and $\mathbb{Z}_{108}$ with the help of a computer search [8]. In this paper we restrict our attention to $N=144$, but we believe that the methods described here can be used to classify all non-periodic tilings for all $N \leq 200$. We also see from Section 4 that the number of non-periodic tilings grows at least exponentially with $N$, so that for large and highly-composite values of $N$ this task gets hopeless. Also, the motivation for musical compositions does not extend beyond $N=200-300$ due to obvious perceptional limitations. Let us also mention that in order to test the Coven-Meyerowitz conditions one will need to list non-periodic tilings of $\mathbb{Z}_{N}$ for values of $N$ containing at least 3 different prime factors, such as $N=120,180,200$, or even much higher.

\section{Deciding the Coven-Meyerowitz Conditions}

In this section we describe in detail an algorithm which, given a set of integers

$$
A \subseteq\{0, \ldots, D\}
$$


decides if $A$ satisfies the $\left(T_{1}\right)$ and $\left(T_{2}\right)$ conditions of Coven and Meyerowitz [5].

Theorem 2.1. There is an algorithm to decide whether conditions $\left(T_{1}\right)$ and $\left(T_{2}\right)$ (see 8 1.3) hold for a given $A \subseteq \mathbb{Z}$ which runs in time polynomial in $D=\operatorname{diam}(A)$. This algorithm therefore decides if $A$ tiles $\mathbb{Z}$ when $|A|$ contains at most two distinct prime factors.

Proof. The algorithms consists of the steps given below.

1. Compute all cyclotomic polynomials of degree up to $D$.

This step is very easy to carry out in time polynomial in $D$ using, for instance, the formula

$$
\Phi_{n}(x)=\prod_{d \mid n}\left(1-x^{n / d}\right)^{\mu(d)},
$$

where $\mu(d)$ is the Möbius function.

2. Determine the cyclotomic divisors of the polynomial $A(x)$.

Again, this is easily doable in time polynomial in $D$. Let $S_{A}$ be the set of indices $n=p_{i}^{a_{i}}$, which are prime powers, such that $\Phi_{n}(x) \mid A(x)$. Let $p_{1}, p_{2}, \ldots p_{k}$ be the different primes whose powers appear here, and let $N_{i} \geq 1, i=1, \ldots, k$, be the number of relevant powers of the prime $p_{i}$.

3. Test if condition $\left(T_{1}\right)$ holds.

Having computed the set $S_{A}$ in the previous step, this amounts to checking the definition of condition $\left(T_{1}\right)$.

4. If $\left(N_{1}+1\right)\left(N_{2}+1\right) \cdots\left(N_{k}+1\right)-1>D$ then answer that $\left(T_{2}\right)$ fails. End.

The explanation of this step is as follows. If $\left(T_{2}\right)$ is to hold then for any choice of prime powers (with respect to different primes) from the set $S_{A}$ there is a different divisor of $A(x)$, namely the cyclotomic polynomial $\Phi_{n}(x)$ where $n$ is the product of the chosen prime powers. Comparing degrees we obtain the inequality

$$
\left(N_{1}+1\right)\left(N_{2}+1\right) \cdots\left(N_{k}+1\right)-1 \leq D .
$$

5. Check exhaustively if $\left(T_{2}\right)$ holds and reply accordingly.

This exhaustive search has to check $\left(N_{1}+1\right)\left(N_{2}+1\right) \cdots\left(N_{k}+1\right)-1$ possibilities for $\left(T_{2}\right)$ to fail (select a power of each involved prime $p_{i}$, with exponent from 0 to $N_{i}$ ). This number is at most $D$ (by the previous step) and therefore the total cost of this step of the algorithm is polynomial in $D$.

The fact that this algorithm decides if $A$ tiles $\mathbb{Z}$ when $|A|$ contains at most two distinct prime factors follows from Theorem B2 in [5] which claims that, in that case, the conjuction $T_{1}$ and $T_{2}$ is equivalent to tiling $\mathbb{Z}$.

\section{The local Coven-Meyerowitz COnditions}

In this section we give a local version of the Coven-Meyerowitz conditions, relevant to tiling a given cyclic group $\mathbb{Z}_{N}$ and not $\mathbb{Z}$, and derive the corresponding properties they enjoy.

Let $N$ be a positive integer. Define $S_{A}^{N}$ be the set of prime powers $p^{a} \mid N$ such that $\Phi_{p^{a}}(X) \mid A(X)$. Similarly define the conditions

$\left(T_{1}^{N}\right): A(1)=\prod_{s \in S_{A}^{N}} \Phi_{s}(1)$,

$\left(T_{2}^{N}\right)$ : If $s_{1}, \ldots, s_{m} \in S_{A}^{N}$ are powers of distinct primes then $\Phi_{s_{1} \cdots s_{m}}(X) \mid A(X)$. 
In Theorem 3.1 below statements (1) and (2) correspond to Theorems A and B1 of [5]. Statement (3) means that the Coven-Meyerowitz conjecture is stronger than the corresponding local conjecture. Statement (4), corresponding to Theorem B2 in [5], means that we know the local condition $T_{2}^{N}$ is also true when at most two primes are involved in $|A|$. Finally, statement (5) means that we have a polynomial time (in $N$ ) algorithm to decide the local Coven-Meyerowitz conditions $T_{1}^{N}$ and $T_{2}^{N}$.

Theorem 3.1. For any finite set $A \subseteq \mathbb{Z}$ and any positive integer $N$ :

(1) $T_{1}^{N}$ and $T_{2}^{N} \Longrightarrow$ A tiles $\mathbb{Z}_{N}$

(2) A tiles $\mathbb{Z}_{N} \Longrightarrow T_{1}^{N}$

(3) If ( $A$ tiles $\mathbb{Z} \Longrightarrow T_{1}$ and $T_{2}$ ) then ( $A$ tiles $\mathbb{Z}_{N} \Longrightarrow T_{1}^{N}$ and $\left.T_{2}^{N}\right)$.

(4) If there are at most two distinct primes in $|A|$ and $A$ tiles $\mathbb{Z}_{N}$ then $T_{1}^{N}$ and $T_{2}^{N}$ hold.

(5) There is an algorithm which decides if $T_{1}^{N}$ and $T_{2}^{N}$ hold in time polynomial in $N$. This algorithm therefore decides if $A$ tiles $\mathbb{Z}_{N}$ when $|A|$ contains at most two distinct prime factors.

Proof. The proof of (1) is essentially the same as that of Theorem A in [5]. When reading that proof it might help to observe that $A(1) B(1)=\operatorname{lcm}\left(S_{A}\right)$, or, in the local version, $A(1) B(1)=\operatorname{lcm}\left(S_{A}^{N}\right)$.

For the proof of $[2]$ we use Lemma 2.1 in [5]. According to that, if $A$ tiles $\mathbb{Z}_{N}$ it follows that $S_{A}$ contains only divisors of $N$. But $A$ tiles $\mathbb{Z}_{N}$ implies that $A$ tiles $\mathbb{Z}$, hence, by Theorem B1 of [5], $T_{1}$ is valid. But $S_{A}=S_{A}^{N}$ in this case hence $T_{1}^{N}$ is valid too.

To prove (3), that the Coven-Meyerowitz conjecture implies the corresponding local conjecture one first observes that if $A$ tiles $\mathbb{Z}_{N}$ then it also tiles $\mathbb{Z}$. By the Coven-Meyerowitz conjecture follows the validity of $T_{1}$ and $T_{2}$ and, finally, observe that $T_{2}$ implies $T_{2}^{N}$ for all $N$.

To show (4) we have, if $A$ tiles $\mathbb{Z}_{N}$, that $A$ tiles $\mathbb{Z}$, hence $T_{2}$ holds (by Theorem B2 in [5]), which implies that $T_{2}^{N}$ holds.

To prove 5 we merely repeat the algorithm of $\$ 2$ but replacing $S_{A}$ by $S_{A}^{N}$, etc. Because of (4) this algorithm also decides if $A$ tiles $\mathbb{Z}_{N}$ if $|A|$ contains at most two distinct prime factors.

\section{The NUMBER OF NON-PERIODIC TILING COMPLEMENTS}

One might expect that the number of non-periodic tilings of $\mathbb{Z}_{N}$ is small. However this is not the case.

Theorem 4.1. There are arbitrarily large $N$ and non-periodic tilings $\mathbb{Z}_{N}=A \oplus B$, such that there are additional distinct non-periodic tiling complements $B_{1}, \ldots, B_{k}$ of $A$, with $k \geq e^{C \sqrt{N}}, C$ a constant.

Proof. The following sketch of a proof relies on a construction given in [17] where it was used to prove that there are tilings of the integers with a tile contained in $\{0, \ldots, D\}$ but of period at least $C D^{2}$.

We take $N=2 \cdot 3 \cdot 5 \cdot p \cdot q$ where $p$ and $q$ are two different large primes, roughly of the same size $\sim \sqrt{N}$. We view the group $\mathbb{Z}_{N}$ as

$$
\mathbb{Z}_{N}=\mathbb{Z}_{3 p} \times \mathbb{Z}_{5 q} \times \mathbb{Z}_{2}
$$

as shown in Fig. 1. We take the set $A$ to be the " $3 \times 5$ rectangle at the origin". The set $A$ can then tile $\mathbb{Z}_{N}$ in a non-periodic way by first tiling the lower and the upper layer in the ordinary way and then perturbing a row of the lower layer and a column of the upper layer, as shown in Fig. 1. Each such perturbation manages to destroy one of the two periods that existed in the ordinary tiling (for a detailed proof see [17]). 


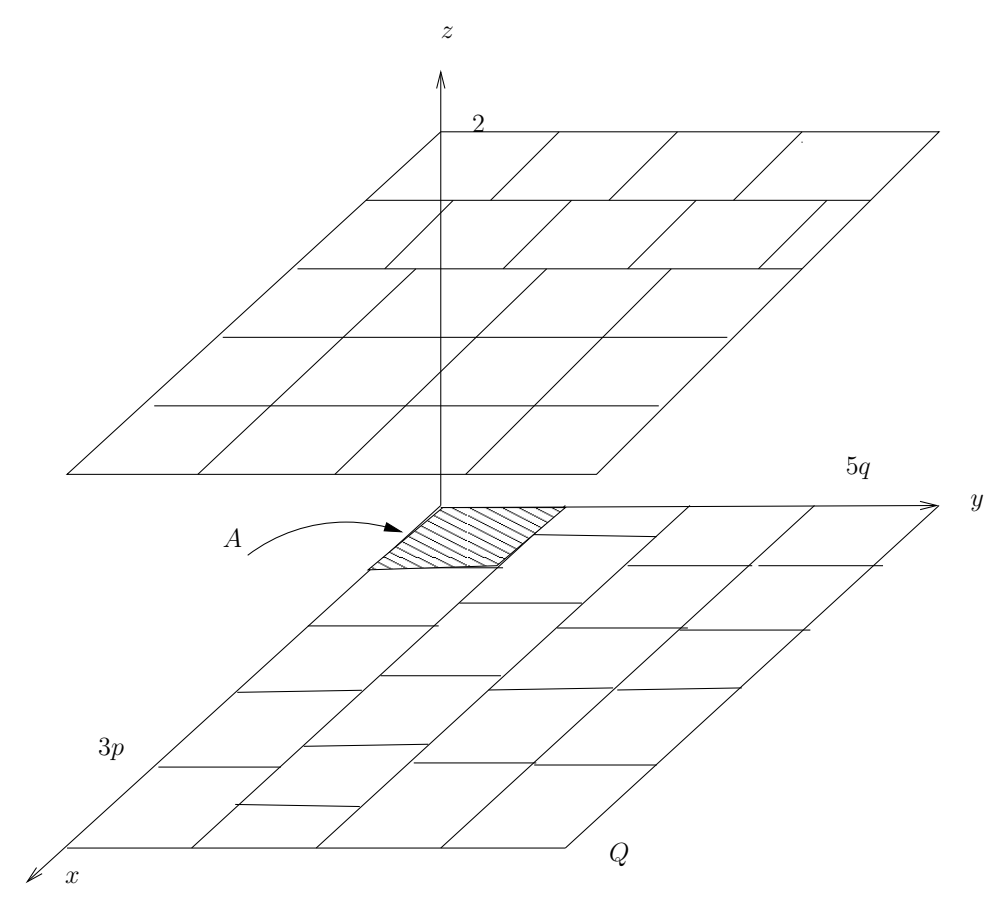

FiguRE 1 . The group $\mathbb{Z}_{3 p} \times \mathbb{Z}_{5 q} \times \mathbb{Z}_{2}$

If one wants to create many different non-periodic tilings of $\mathbb{Z}_{N}$ with $A$ one does as follows.

(1) Keep the upper layer the same (with one perturbed row only).

(2) Keep half the lower layer the same: rows indexed from $5 q / 2$ to $5 q$ are left unperturbed (refer to Fig. 1).

(3) Perturb the remaining rows of the lower layer arbitrarily, subject only to the restriction that at least one of them is perturbed by a non-zero amount.

It is clear that the number of such complements is at least

$$
\sim 3^{5 q / 2} \sim e^{C \sqrt{N}} .
$$

Each such tiling is non-periodic: suppose $g=(a, b, c) \in \mathbb{Z}_{3 p} \times \mathbb{Z}_{5 q} \times \mathbb{Z}_{2}$ is a period. The two layers cannot be swapped by a translation by $g$, as one has perturbed rows but the other one does not, so $c=0$. The upper layer is mapped to itself by a $g$-translation. This can only happen if $b=0$ as the perturbed column is unique. Finally $a=0$ since the lower layer cannot move in the direction of $\mathbb{Z}_{5 q}$ and be mapped into itself: the perturbed row closest (in the "direction of motion") to the unperturbed block of $\sim 5 q / 2$ rows cannot move to a perturbed row.

Even if one considers two tilings which differ by a translation identical the number would still remain exponential in $N$.

\section{Finding ALL NON-PERIODIC TILINGS IN A CYCLIC GROUP}

As explained in Section 1 there is a particular motivation coming from music compositions to list out all non-periodic tilings of a cyclic group $\mathbb{Z}_{N}$, for relatively small values of $N$. H. Fripertinger [8] achieved this task for $N=72,108$. In this section we settle $N=144$, and we believe that the algorithms described here are likely to work for other values like $N=120,180,200,216$. On the other hand we see from Theorem 4.1 that this task becomes hopeless for large and "highly composite" $N$. 
We cannot offer any rigorous mathematical statements here with regard to the complexity of the algorithms used. We provide heuristics, observe that methods seem to work remarkably well and we are able to achieve a full classification for $N=144$.

Remark 5.1. Throughout this section we rely on results of [5]. Our algorithm works only when tiling implies the $T_{2}^{N}$ condition (see §3). In particular it works when either $N$ has at most two distinct prime factors, such as $N=144=2^{4} \cdot 3^{2}$, or has three distinct prime factors but one of them appears to the first power, such as $N=120=2^{3} \cdot 3 \cdot 5$ or $N=180=2^{2} \cdot 3^{2} \cdot 5$. The reason for this is that in any tiling $\mathbb{Z}_{N}=A \oplus B$ in such a case either $|A|$ or $|B|$ has at most two distinct primes factors.

A basic ingredient of our algorithm is the "fill-out procedure" performing the following task: given a set $A$ in a group $G$, list out all tiling complements $B$ of $A$. We cannot give any precise statement on how efficiently this task can be achieved except to note that it performs really well in practice. However, let us mention here that the method described below was already successfully used in [6] in refuting a conjecture of Lagarias and Szabó on a necessary condition for the existence of universal spectrum, and it could well be useful in tackling other question related to tiling. The credit for this heuristic algorithm goes to P. Móra in [6].

\section{The Fill-out Procedure.}

Assume $A \subset \mathbb{Z}_{N}$ is given (in fact, the procedure works for any finite abelian group $G$, but we restrict our attention to cyclic groups). The task is to find all tiling complements $B$ of $A$. As usual, we make the normalization assumption that $0 \in A, B$.

We will build up, by adding elements one by one, all tiling complements $B$ of $A$. For this, we move in the space of packing complements $P$ of $A$, i.e. sets $P=\left\{p_{1}, \ldots p_{m}\right\}$ such that all translated copies $A+p_{j}$ are disjoint from each other. We are trying to grow the sets $P$ so that they become tiling complements. Our exploration starts with the set $P=\{0\}$. At any given step we will attempt to extend $P$ by one new element in a particular manner (which is described in detail in the next paragraph). If no such element exists we backtrack. We describe our algorithm "explore" below, and note that explore $(P)$ finds all tiling complements $B$ containing $P$. We invoke it as explore $(\{0\})$ with the list of tiling complements of $A$ being initially empty. The recursive procedure explore is described in Fig. 2,

The efficiency of this procedure is due to the heuristic of expanding a given packing complement $P$ of $A$ by adding to it a copy of $A$ which covers an element $x$ that is the most restrictive in the sense that there are few possible ways to cover it. This is achieved by ranking the yet uncovered elements $x$ in increasing order of the function $r(x)=r(P, x)$ that counts in how many ways an element $x$ can be covered by adding one more copy of $A$ to the given packing $P$. Clearly, if $r(x)=0$ for some $x$ then $x$ cannot be covered in any admissible way, so that $P$ can definitely not be extended to a tiling complement (it is still possible that $P$ could be extended by several further elements to form a larger packing complement $P^{\prime}$, but it can never grow to become a tiling complement due to $x$ never being covered). It also often happens that $r(x)=1$, i.e. we are forced to place the next copy of $A$ at a certain position in order to cover $x$, and once this copy is placed, there is even less room in $\mathbb{Z}_{N}$, and it is likely that there will be another $x$ such that $r(x)=1$, or at least small.

With this fill-out procedure at hand we can now describe the algorithm to list all nonperiodic tilings of $\mathbb{Z}_{144}$. We merely describe the algorithm here, as the full documentation (or listing out the numerous arising sets) would be far too lengthy for this paper. The results and documentation are available online at [16]. 
explore $(P)$

(1) If $P$ has already been explored, return.

(2) Mark $P$ as explored.

(3) If $P$ is a tiling complement of $A$, add it to the list of tiling complements and return.

(4) Compute the function $r: \mathbb{Z}_{N} \backslash(A \oplus P) \rightarrow \mathbb{N}$, defined by $r(x)=$ number of ways to add an extra copy of $A$ to $A \oplus P$ so that $x$ is now covered and the new copy of $A$ does not intersect $A \oplus P$.

(5) Rank all $x \in \mathbb{Z}_{N} \backslash(A \oplus P)$ according to $r(x)$ :

$$
r\left(x_{1}\right) \leq r\left(x_{2}\right) \leq \cdots \leq r\left(x_{k}\right),
$$

in such a way that if $r\left(x_{i}\right)=r\left(x_{i+1}\right)$ then $x_{i}<x_{i+1}$ (for this purpose of ordering the elements of $\mathbb{Z}_{N}$ are thought of as $\left.\{0,1, \ldots N-1\}\right)$. Here $k=\left|\mathbb{Z}_{N} \backslash(A \oplus P)\right|$.

(6) If $r\left(x_{1}\right)=0$, return.

(7) Consider $x_{1}$.

- Let $A+y_{1}, \ldots, A+y_{r\left(x_{1}\right)}$ be the copies of $A$ that can be added to $A \oplus P$ in a non-overlapping way and that contain $x_{1}$.

- explore $\left(P \cup\left\{y_{1}\right\}\right), \ldots$, explore $\left(P \cup\left\{y_{x\left(r_{1}\right)}\right\}\right)$.

FiguRE 2. The explore recursive procedure

Normalizing conditions. We always assume that $0 \in A, B$. Also, it is clear that in terms of tiling questions a set $A=\left\{0, a_{1}, a_{2}, \ldots\right\}$ is equivalent to its own translated copies $A-a_{1}, A-a_{2}, \ldots$ all containing zero. It is customary to include only one representative from these equivalent copies, and we are going to follow this tradition (so that the numbers described in the last section correspond to this normalization).

\section{The Algorithm.}

Step 1. The prime powers dividing 144 are $2,4,8,16,3$ and 9 . In any tiling $A \oplus B=\mathbb{Z}_{144}$ the cyclotomic polynomials corresponding to these prime powers must divide exactly one of $A(x)$ and $B(x)$, according to condition $T_{1}$ of [5]. Therefore we first make a list of all possible partitions $\left\{H, H^{c}\right\}$ of the elements $\{2,4,8,16,3,9\}$. There are 32 such partitions (note that $\left\{H, H^{c}\right\}$ and $\left\{H^{c}, H\right\}$ are the same). Our task is to decide for each partition whether any non-periodic tilings correspond to it.

Step 2. Certain partitions produce only periodic tilings due to condition $T_{2}$ of [5]. Indeed, as an example, take the partition $\{\{2,4\},\{8,16,3,9\}\}$. Due to condition $T_{2}$ of [5] the cyclotomic polynomials $\Phi_{24}(x), \Phi_{72}(x), \Phi_{48}(x), \Phi_{144}(x)$ must also divide $B(x)$, together with $\Phi_{8}(x), \Phi_{16}(x), \Phi_{3}(x), \Phi_{9}(x)$. From this it follows that the support of $\widehat{\chi_{B}}$ is contained in the subgroup $\{0,2,4, \ldots, 142\}$ and this automatically makes $B$ periodic. Therefore, in this step, discard all partitions which imply automatic periodicity of either $A$ or $B$ by condition $T_{2}$.

The remaining partitions are:

$\{\{3,4,8\},\{2,9,16\}\},\{\{3,4,9\},\{2,8,16\}\},\{\{3,4,16\},\{2,8,9\}\},\{\{3,8,9\},\{2,4,16\}\}$, $\{\{3,8,16\},\{2,4,9\}\},\{\{4,8,9\},\{2,3,16\}\},\{\{4,8,16\},\{2,3,9\}\},\{\{4,9,16\},\{2,3,8\}\}$, $\{\{8,9,16\},\{2,3,4\}\},\{\{2,3,4,8\},\{9,16\}\},\{\{2,3,4,16\},\{8,9\}\},\{\{2,3,8,9\},\{4,16\}\}$, $\{\{2,3,8,16\},\{4,9\}\},\{\{2,4,8,16\},\{3,9\}\},\{\{2,4,9,16\},\{3,8\}\},\{\{2,8,9,16\},\{3,4\}\}$, $\{\{3,4,8,16\},\{2,9\}\},\{\{4,8,9,16\},\{2,3\}\},\{\{2,3,4,8,16\},\{9\}\},\{\{3,4,8,9\},\{2,16\}\}$, $\{\{2,3,4,9\},\{8,16\}\},\{\{2,3,4,8,9\},\{16\}\}$. 
We remark here that most of these partitions will not produce non-periodic tilings. If there were further theoretical considerations upon which certain partitions could be discarded, then it would probably be possible to apply our algorithm for higher values of $N$. For instance, it could be true that if either $|A|$ or $|B|$ is a prime power then the tiling must be periodic. This would allow us to discard several further partitions.

Assume we are dealing with a fixed partition $P=\left\{H, H^{c}\right\}$. The natural approach is the following: by the structure theory given in Section 4 of [5] one can list out all subsets $A \subset \mathbb{Z}_{144}$ such that $A$ tiles $\mathbb{Z}_{144}$ and $\Phi_{h}(x)$ divides $A(x)$ for all $h \in H$.

We remark here that besides using the structure theory of 5 there is an alternative way of listing out these sets $A$. Namely, take the least common multiple $L$ of the prime powers appearing in $H$. By the Remark following the proof of Theorem A in [5] one can construct a universal tiling complement $B$ in $\mathbb{Z}_{L}$ of any tile $C$ in $\mathbb{Z}_{L}$ such that $\Phi_{h}(x)$ divides $C(x)$ for all $h \in H$. The set $B$ is constructed by taking the product of certain cyclotomic polynomials as described in the proof of Theorem A of [5]. Next we apply our fill-out procedure to $B$ in $\mathbb{Z}_{L}$, and hence list out all possible sets $C$ in $\mathbb{Z}_{L}$. Finally, all the desired tiles $A$ in $\mathbb{Z}_{N}$ must reduce to one of these sets $C$ modulo $L$. Therefore, to list out the sets $A$ we must shift the elements of sets $C$ by multiples of $L$ in all possible ways.

Then these sets $A$ need to be grouped into equivalence classes according to the zero set of the Fourier transform of $\chi_{A}$. A similar procedure must be done for subsets $B$. Then one needs to select the equivalence classes which correspond to non-periodic sets $A$ and $B$ (this can be read off from the zero-sets of the Fourier transform, or directly from any particular representative of the class). Finally, if a class of non-periodic $A$ 's and a class of non-periodic $B$ 's are such that the union of the zero-sets of their Fourier transform contains $\mathbb{Z}_{144} \backslash\{0\}$, then any representatives of these classes tile with each other in a non-periodic way.

For some partitions this approach can indeed be carried out. However, the problem is that for certain partitions the number of tiles $A$ and/or $B$ provided by the structure theory of [5] can be overwhelmingly large. Take the example $\{\{2,3,8,16\},\{4,9\}\}$. The structure theory of [5] provides two different tiles $A_{1}, A_{2}$ of cardinality 24 modulo $\operatorname{lcm}(2,3,8,16)=$ 48 , such that $A(x)$ is divisible by each of $\Phi_{\{2,3,8,16\}}(x)$. The tiles $A$ modulo 144 are therefore obtained as sets that reduce to $A_{1}$ or $A_{2}$ modulo 48 . There are $2 \cdot 3^{23}$ such sets.

Step 3. To overcome this problem, we only list one type of the tiles (either $A$ 's or $B$ 's, whichever are fewer). In the above example we list the tiles of type $B$. The structure theory of [5] provides six different tiles $B_{1}, \ldots B_{6}$ of cardinality 6 modulo $\operatorname{lcm}(4,9)=36$, such that $B(x)$ is divisible by each of $\Phi_{\{4,9\}}(x)$. The tiles $B$ modulo 144 are therefore obtained as sets that reduce to any of $B_{1}, \ldots B_{6}$ modulo 36 . There are $6 \cdot 4^{5}=6144$ such sets. We can easily sort them into equivalence classes according to the zero-set of their Fourier transforms. There are five classes $C_{1}, \ldots, C_{5}$ corresponding to non-periodic tiles $B$. They are characterized by the cyclotomic divisors $\Phi_{h}(x)$, with $h$ being in $C_{1}=\{72,36,18,9,4\}$, $C_{2}=\{36,12,9,4\}, C_{3}=\{36,18,9,4\}, C_{4}=\{36,18,12,9,4\}, C_{5}=\{72,36,18,12,9,4\}$.

Step 4. Next we discard those equivalence classes $C_{j}$ which automatically make any tiling complement $A$ periodic. Consider, for example, $C_{1}$ above so that our tile $B$ has cyclotomic divisors $\Phi_{\{72,36,18,9,4\}}$. Then any tiling complement $A$ of $B$ must have cyclotomic divisors $\Phi_{\{2,3,6,8,12,16,24,48,144\}}$ (and possibly others), which automatically make $A$ periodic.

Step 5. For any remaining equivalence class $C_{j}$ we take a representative $B_{j}$, and run our "fill-out procedure" to find all tiling complements $A$ of $B_{j}$. The point is that $B_{j}$ is non-periodic, so that heuristically we expect not too many tiling complements of $B_{j}$ to 
exist (although this heuristic breaks down if $N$ is large and highly composite, as shown by Theorem 4.1). Once we have all tiling complements $A$ of $B_{j}$ we can select the nonperiodic ones, if any. Let $N_{j}$ denote the collection of all non-periodic tiling complements $A$ of $B_{j}$. Then we conclude that all sets $A$ in $N_{j}$ tile with all representatives $B$ of the class $C_{j}$. We repeat this procedure for all $j$ and we arrive at a list of all non-periodic tilings corresponding to the fixed partition $P$. In the specific example above, Step 4 already discards all classes $C_{j}$ so that Step 5 becomes unnecessary.

We repeat Steps 3-5 for all partitions $P$ listed in Step 2, and we arrive at a list of all non-periodic tilings of $\mathbb{Z}_{144}$.

As a last normalization step, we must recall that a tile $A=\left\{0, a_{1}, \ldots\right\}$ and its shifted copies $A-a_{1}$, etc. are considered equivalent, and keep only one representative for each tile. And, naturally, the same applies to the tiles of type $B$.

Step 6. Exceptional cases. We have been able to execute the steps above for all partitions listed in Step 2 except for $\{\{2,4,8,16\},\{3,9\}\}$. In that case the number of tiles provided by the structure theory of [5] is too large for both $A$ and $B$, and therefore the numerical search would take several days. We choose to deal with this case by invoking some results from the literature. We will show that either $A$ or $B$ must be periodic.

Note that $|A|=16$ and $|B|=9$ by condition $T_{1}$ of [5]. Recall the following.

Theorem 5.2. (Sands [25]) If $A \oplus B=\mathbb{Z}_{N}$ and $N$ has at most two prime factors $p, q$ then either $A$ or $B$ is contained in the subgroup $p \mathbb{Z}_{N}$ or $q \mathbb{Z}_{N}$.

Assume $|A|$ is contained in a subgroup. Then the subgroup must be $H_{3}=\{0,3,6, \ldots 144\}$ due to obvious cardinality reasons. Then $B$ is a union of three parts $B_{0}, B_{1}, B_{2}$ according to residues modulo 3. All three parts must have cardinality 3 , and $A \oplus B_{0}$ gives a tiling of the subgroup $H_{3}$. Note that $H_{3}$ is isomorphic to $\mathbb{Z}_{48}$. If $A$ is not periodic then $B_{0}$ must be periodic (all tilings of $\mathbb{Z}_{48}$ are periodic). Therefore we must have $B_{0}=\{0,48,96\}$. Similarly, $B_{1}$ and $B_{2}$ must be periodic by 48 , and therefore so is $B$.

Assume now that $B$ is contained in a subgroup. Then the subgroup must be $H_{2}=$ $\{0,2,4, \ldots, 144\}$. Then $A$ is a union of $A_{0}$ and $A_{1}$, the even and odd parts of $A$, and $A_{0} \oplus B=H_{2}$. Note that $H_{2}$ is isomorphic to $\mathbb{Z}_{72}$. If $B$ is non-periodic then $A_{0}$ must be periodic (the only non-periodic tilings of $\mathbb{Z}_{72}$ contain tiles of cardinality 6 and 12 , while $|B|=9$ and $\left.\left|A_{0}\right|=8\right)$. In fact, $A_{0}$ must be periodic by 72 , because the periodicity subgroup contained in $A_{0}$ must divide the cardinality $\left|A_{0}\right|=8$. By the same reasoning we obtain that $A_{1}$ is also periodic by 72 , and therefore so is $A$.

Summary of the results for $\mathbb{Z}_{144}$.

For the partition $\{\{2,8,9,16\},\{3,4\}\}$ there is a set $T_{1}$ containing 36 non-periodic tiles of cardinality 24 (type $A$ ), and a set $T_{2}$ containing 6 non-periodic tiles of cardinality 6 (type $B$ ). Each tile in $T_{1}$ tiles with each in $T_{2}$. The 36 tiles in $T_{1}$ fall into two different equivalence classes corresponding to the following cyclotomic factors: $\Phi_{\{144,72,24,18,16,9,8,2\}}$, and $\Phi_{\{144,72,18,16,9,8,2\}}$. The 6 tiles in $T_{2}$ fall into one equivalence class corresponding to the cyclotomic factors $\Phi_{\{48,36,24,12,6,4,3\}}$. We present here one representative for $T_{1}$ and $T_{2}$ (one can then easily recover the full sets by applying the "fill-out procedure" to these representatives):

- $\{0,17,20,23,28,29,40,48,53,59,65,68,76,88,89,95,96,101,116,124,125$, $131,136,137\}$ is in $T_{1}$ and

- $\{0,32,58,90,112,122\}$ is in $T_{2}$. 
For the partition $\{\{4,9,16\},\{2,3,8\}\}$ there is a set $T_{1}$ containing 6 non-periodic tiles of cardinality 12 (type $A$ ), and a set $T_{2}$ containing 324 non-periodic tiles of cardinality 12 (type $B$ ). Each tile in $T_{1}$ tiles with each in $T_{2}$. The 6 tiles in $T_{1}$ fall into one equivalence class corresponding to the following cyclotomic factors: $\Phi_{\{144,36,18,16,9,4\}}$. The 324 tiles in $T_{2}$ fall into two equivalence classes corresponding to the cyclotomic factors $\Phi_{\{72,48,24,18,12,8,6,3,2\}}$, and $\Phi_{\{72,48,24,12,8,6,3,2\}}$. We present here one representative for $T_{1}$ and $T_{2}$ (one can then easily recover the full sets by applying the "fill-out procedure" to these representatives):

- $\{0,34,40,46,48,58,88,96,106,118,130,136\}$ is in $T_{1}$ and

- $\{0,16,29,44,57,73,80,93,108,109,124,137\}$ is in $T_{2}$.

For the partition $\{\{2,4,9,16\},\{3,8\}\}$ there is a set $T_{1}$ containing 8640 non-periodic tiles of cardinality 24 (type $A$ ), and a set $T_{2}$ containing 3 non-periodic tiles of cardinality 6 (type $B$ ). Each tile in $T_{1}$ tiles with each in $T_{2}$. The 8640 tiles in $T_{1}$ fall into three different equivalence classes corresponding to the following cyclotomic factors: $\Phi_{\{144,36,18,16,12,9,4,2\}}$, $\Phi_{\{144,36,18,16,9,6,4,2\}}$, and $\Phi_{\{144,36,18,16,9,4,2\}}$. The 3 tiles in $T_{2}$ fall into one equivalence class corresponding to the cyclotomic factors $\Phi_{\{72,48,24,12,8,6,3\}}$ We present here one representative for $T_{1}$ and $T_{2}$ (one can then easily recover the full sets by applying the "fill-out procedure" to these representatives):

- $\{0,9,17,26,27,34,39,40,48,51,57,65,74,82,88,96,99,105,111,113,122$, $123,130,136\}$ is in $T_{1}$ and

- $\{0,36,64,80,100,116\}$ is in $T_{2}$.

The partition $\{\{3,4,8\},\{2,9,16\}\}$ is the most interesting. There are sets $T_{1}$ and $S_{1}$ containing respectively 6 and 156 non-periodic tiles of cardinality 12 (type $A$ ), corresponding to the cyclotomic factors $\Phi_{\{72,48,36,24,12,8,6,4,3\}}$ and $\Phi_{\{72,48,24,12,8,6,4,3\}}$, respectively. Also, there are sets $T_{2}$ and $S_{2}$ containing respectively 12 and 48 non-periodic tiles of cardinality 12 (type $B$ ), corresponding to the cyclotomic factors $\Phi_{\{144,36,18,16,9,2\}}$ and $\Phi_{\{144,18,16,9,2\}}$, respectively. Sets in $T_{1}$ tile with all sets in both $T_{2}$ and $S_{2}$. Sets in $S_{1}$ only tile with sets in $T_{2}$. We give a representative for each of $T_{1}, S_{1}, T_{2}, S_{2}$ :

- $\{0,18,28,44,54,64,80,82,98,108,118,134\}$ is in $T_{1}$,

- $\{0,16,30,44,58,74,80,94,108,110,124,138\}$ is in $S_{1}$,

- $\{0,33,40,45,48,57,88,96,105,117,129,136\}$ is in $T_{2}$, and

- $\{0,27,30,35,60,72,75,83,102,123,131,132\}$ is in $S_{2}$.

The other partitions listed in Step 2 do not produce non-periodic tilings.

\section{REFERENCES}

[1] M. Andreatta, C. Agon, E. Amiot, Tiling problems in music composition: Theory and Implementation, International Computer Music Conference, Göteborg, 2002, 156-163.

[2] E. Amiot, Why rhythmic Canons are interesting, Perspectives in Mathematical and Computational Music Theory, EpOs, 194-213, 2004.

[3] M. Andreatta, On group-theoretical methods applied to music: some compositional and implementational aspects. Perspectives in Mathematical and Computational Music Theory, EpOs, 169-193, 2004.

[4] R. Berger, The undecidability of the domino problem, Memoirs of the Amer. Math. Soc. 66 (1966), $1-72$.

[5] E. Coven and A. Meyerowitz, Tiling the integers with translates of one finite set, J. Algebra 212 (1999), 1, 161-174.

[6] B. Farkas, M. Matolcsi, P. Móra: On Fuglede's conjecture and the existence of universal spectra, J. Fourier Anal. Appl., Volume 12, Number 5, (2006), 483-494.

[7] H. Fripertinger, Tilings Problems in Music Theory, Perspectives in Mathematical and Computational Music Theory, EpOs, 153-168, 2004. 
[8] H. Fripertinger, Classification of rhythmical canons, at http://www .mathe2.uni-bayreuth.de/frib/canons/canon.html

[9] B. Fuglede, Commuting self-adjoint partial differential operators and a group theoretic problem, J. Funct. Anal. 16 (1974), 101-121.

[10] D. Girault-Beauquier, M. Nivat, Tiling the plane with one tile, in: Topology and Category Theory in Computer Science, G.M. Reed, A.W. Roscoe, R.F. Wachter (eds.), Oxford Univ. Press 1989, 291-333.

[11] B. Grünbaum and G.C. Shepard, Tilings and patterns, New York: Freeman 1987.

[12] G. Hajós, Sur la factorization des groupes abéliens, Casopis Pest Mat. Fys. 74 (1950), 157-162.

[13] M.N. Kolountzakis and M. Matolcsi, Complex Hadamard matrices and the spectral set conjecture, Collect. Math. (2006), Vol. Extra, 281-291.

[14] S. Konyagin and I. Eaba, Spectra of certain types of polynomials and tiling of integers with translates of finite sets, J. Number Th. 103 (2003), 2, 267-280.

[15] M.N. Kolountzakis, The study of translational tiling with Fourier Analysis, in Fourier Analysis and Convexity, 131-187, Appl. Numer. Harmon. Anal., Birkhäuser Boston, Boston, MA, 2004.

[16] M.N. Kolountzakis, M. Matolcsi, The documentation of non-periodic tilings modulo 144, at http://fourier.math.uoc.gr/ ${ }^{\sim m k} /$ tiling-computations\}.

[17] M.N. Kolountzakis, Translational tilings of the integers with long periods, Electr. J. Combinatorics 10 (2003), 1, R22.

[18] J. C. Lagarias and Y. Wang, Tiling the line with translates of one tile, Inventiones Math. 124 (1996), 341-365.

[19] J.C. Lagarias and Y. Wang, Spectral sets and factorizations of finite abelian groups, J. Funct. Anal. 145 (1997),73-98.

[20] D.J. Newman, Tesselations of integers, J. Number Th. 9 (1977), pp. 107-111.

[21] R.M. Robinson, Undecidability and nonperiodicity for tilings of the plane, Invent. Math. 12 (1971), 177-209.

[22] W. Rudin, Fourier analysis on groups, Reprint of the 1962 original. Wiley Classics Library. A WileyInterscience Publication. John Wiley \& Sons, Inc., New York, 1990.

[23] A.D. Sands, On the factorization of finite Abelian groups, Acta Math. Acad Sci. Hungar. 8 (1957), 65-86.

[24] A.D. Sands, On the factorization of finite Abelian groups II, Acta Math. Acad Sci. Hungar. 13 (1962), 153-159.

[25] A.D. Sands, On Keller's conjecture for certain cyclic groups, Proc. Edinburgh Math. Soc. (2), 22, (1977), 17-21.

[26] M. Szegedy, Algorithms to tile the infinite grid with finite clusters, In Proceedings of the 39th Annual Symposium on the Foundations of Computer Science 1998, 137-145.

[27] D.T. Vuza, Supplementary Sets and Regular Complementary Unending Canons, Perspectives of New Music, nos 29(2) 22-49; 30(1), 184-207; 30(2), 102-125; 31(1), 270-305. 1991.

[28] H.A.G. Wijshoff and J. van Leeuwen, Arbitrary versus periodic storage schemes and tesselations of the plane using one type of polyomino, Information and Control 62 (1984), 1-25.

M.K.: Department of Mathematics, University of Crete, Knossos Ave., GR-714 09, Iraklio, GREECE

E-mail address: kolount@gmail.com

M.M.: Alfréd Rényi Institute of Mathematics, Hungarian Academy of Sciences POB 127 H-1364 Budapest, Hungary.

(also at BME Department of Analysis, Budapest, H-1111, Egry J. U. 1)

E-mail address: matomate@renyi.hu 